\title{
Haptics democratization: challenges and opportunities
}

\author{
Robert Iacob $^{1 *}$, and Diana Popescu ${ }^{1}$ \\ ${ }^{1}$ University Politehnica of Bucharest, Robotics and Manufacturing Systems Department, \\ Splaiul Independenței nr. 313, București, România - RO:060042
}

\begin{abstract}
The sense of touch is an important means of communicating and exchanging information primarily between people and between people and the environment, from the very first years of life. Nowadays, researches and technology advances in different domains make use of touch, man-computer communication and interaction throughout immersive simulation environments being also facilitated and enhanced by the kinesthetic technology.

The importance of haptic feedback for different applications is already a proved fact. However, it is still underrepresented in the everyday computer interfaces, mainly due to a current unfavourable compromise between price and user's experience quality. That is, even if many haptic devices are available, they are either prohibitive in term of price, if provide high quality, or they are inexpensive and offer low realism haptic effects. Currently, there is no real intermediate device, and no device that can provide hardware upgrade according to users' needs.

In this context, this paper provides an analysis of existing haptic equipment, both prototypes and commercially available devices, and presents different approaches used in haptic-based scenarios. On this basis, a critical set of requirements for a new type of haptic device are inferred. It could represent an innovative and affordable solution for a larger range of potential users: engineers, physicists, trainers, designers, researchers, as well as hobbyists.
\end{abstract}

\section{Introduction}

A comprehensive definition of Virtual Reality (VR) should mandatory refer to and explain the link between technological hardware and human experience. Gibson considers the concept of presence as relevant for the virtual human experience, defining it as "the sense of being in an environment", and relates it with the way the user perceives the virtual "surroundings as mediated by both automatic and controlled mental processes" [1]. The sense of presence involves the use of human sensory channels, which provides information processed by the brain along with other information from the experiences previously acquired from the interaction with the real world.

\footnotetext{
${ }^{*}$ Corresponding author: robert.iacob@gmail.com
} 
It was proven by Robles de la Torre that the sense of touch has a crucial role in the real life, force-feedback information about the environment being used to control the body movements, to gauge the properties of objects or to perceive the world. By extension, a similar, very important, role is played by the sense of touch in the Virtual Environment (VE). Thus, if a global interface is not offering adequate information about the environment, it could be impossible for users to perform different tasks and to be fully immersed in the VE [2].

According to Burdea [3], haptic technology or haptics is a virtual type of sensorial interaction which groups the modalities of:

$\square$ force feedback - providing information on a virtual object hardness, weight, inertia;

$\square$ tactile feedback - giving data about the object surface, smoothness and temperature.

This technology is based on understanding how the human sense of touch works, in order to be able to create virtual objects that can be haptic controlled by the user with the support of dedicated equipment. Therefore, haptic technology can represent, in some way, the third dimension of any immersive platform.

For an efficient simulation environment, a perfect integration between hardware and software is necessary. And although haptic technology has been used for some time, it is generally based on vibrations for products widely used, like: mobile phones, game consoles, car navigation systems etc., or on highly complex feedback mechanisms in case of the devices deployed in medicine and engineering fields. It is important to mention that the first category of products has affordable prices, but they offer an extremely low haptic effect, while the second category of devices offers accurate haptic effects, but the associated price is extremely high. Therefore, a set of high-quality equipment to stimulate the senses from a digital world becomes a mandatory condition and it should take the form of an affordable, reliable and adaptable haptic interface, able to cope with the requirements of different users from companies, universities, research laboratories, especially when used for applications from the mechanical engineering field.

This research uses a standard classification of haptic interfaces in two main categories:

$\square$ basic-haptic - offering limited haptic effect through a "vibro" or "thermo" method;

$\square$ complete-haptic - offering complete haptic effect generated through a frame-based system.

In this context of research on haptic technology, this paper contains, in the second section, an analysis of some basic-haptic devices followed, in the third section, by a critical examination of several complete-haptic equipment developed in-house by researchers or commercialized by different producers. A list of current gaps in haptic technology is identified and detailed in the fourth section. This list represents the basis for setting the design requirements of a new type of intelligent and affordable haptic device.

\section{Basic-haptic devices}

Currently, the most used tactile technology is deployed through the vibration effect. Almost all mobile phones and communicating devices offer now simple vibrating modes. Other inexpensive devices that use the same technology are the game interfaces like: Logitech Flight System, AVB Racing Wheel, Saitek Cyborg Evo Joystick or Nintendo Wii. They provide a basic haptic effect, at a very low price.

A similar category of devices that uses the tactile feedback effect is represented by computer mouse. An example is the Logitech Wingman Force Feedback mouse, based on a technology licensed by Immersion Corporation (FEELit Mouse - the first tactile mouse developed) allowing the user to feel the surfaces of simulated objects. A different version was developed by Bradford Paley [4]. The invention refers to a 3D mouse which includes hand-held housing, adapted for use in space (Figure 1). 


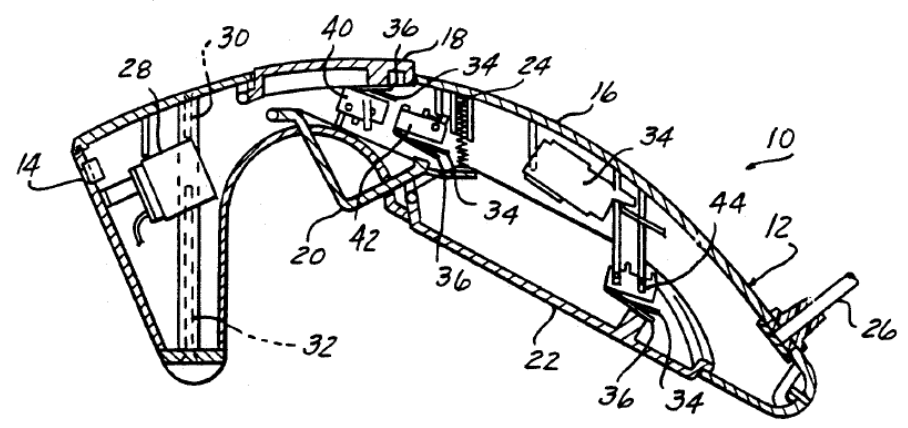

Fig. 1. 3D Tactile Mouse [04]

In order to offer a simple force feedback, Microsoft (USA) proposed a mechanism (Figure 2.) that can be integrated in a gamepad device [5]. The input device includes a mass and a shaft driven by an electric motor. When the mass is accelerated or stopped, the user experiences a simple haptic force.

These types of devices have really low prices - around 100 euro, but they offer extremely low haptic feedback. Having a price far more elevated - around 10.000 euro, the tactile gloves are more appropriate when the VR simulation requires dexterity, i.e. multiple points of contact, freedom of motion, and information about the object grasping state and its mechanical texture [6]. A well-known example is the commercial CyberTouch produced by CyberGlove Systems, a glove including electromechanical vibrators. A similar device, named GhostGlove that delivers haptic sensations to the entire hand, was designed by Tachi Lab, but only for research purposes. A team of engineers from the Imperial College London developed a Vibrating Teaching Glove that uses a combination of vibrating pads and sensors to detect the twisting and flexing of a person's joints in order to help guide the user movements for different sports. The proposed glove can download the movements of popular sports stars and then it can teach the user how to mimic these movements. More recently, a new type of haptic glove was proposed by Oculus VR (Figure 3). The device contains an actuator coupled to the glove body and to the first and second tendons (digits), the actuator being configured to actuate the tendons to control the movement of the glove digit [7].

The existing tactile gloves offer a set of limited haptic effects for a high price, which heavily limits their spread. Moreover, their further integration in a complex immersive system is quite difficult.
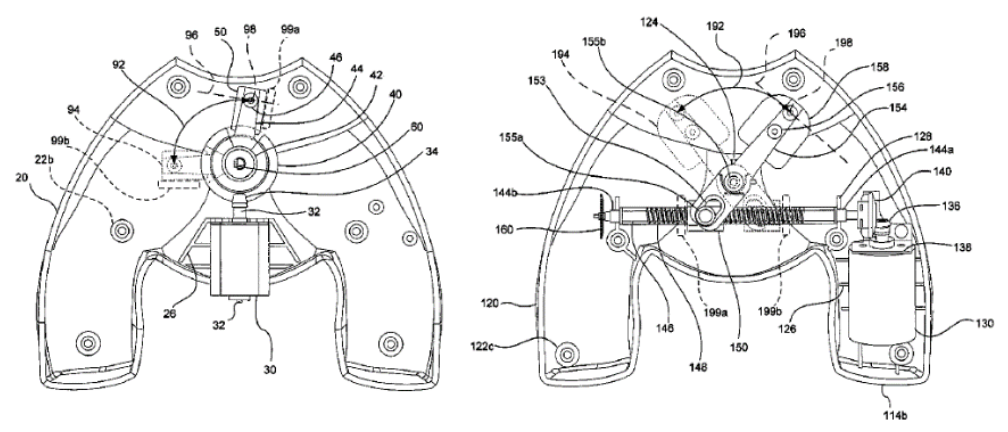

Fig. 2. Microsoft force feedback mechanism [05] 


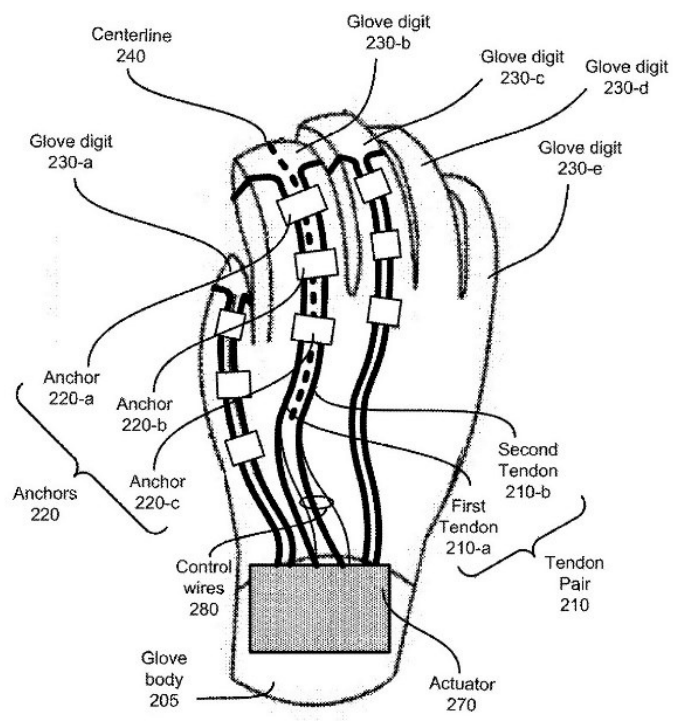

Fig. 3. Oculus VR haptic glove [07]

The tactile feedback research domain is very dynamic, continuously offering interesting approaches and devices. Nintendo is developing a silicon pad, named AFGT that uses tactility to enhance video gaming experience. It is made up of touch-sensitive silicon pockets allowing the user to feel a VE. Another tactile interface is the Sense device currently under development. It is a sensitive interface designed to create a tactile experience of the physical, thermal and haptic properties of products. It integrates a smell magnification system based on the five fundamental flavors and seven fundamental smells. A new level of haptic interaction is proposed by Nokia - the vibrating tattoo [8]. They propose a haptic tattoo based on ferromagnetic ink, able to transmit a 'perceivable' impulse through the skin when a person gets a call, a message or any other alert. More, through this tattoo, one can even get in touch with some simple customization options.

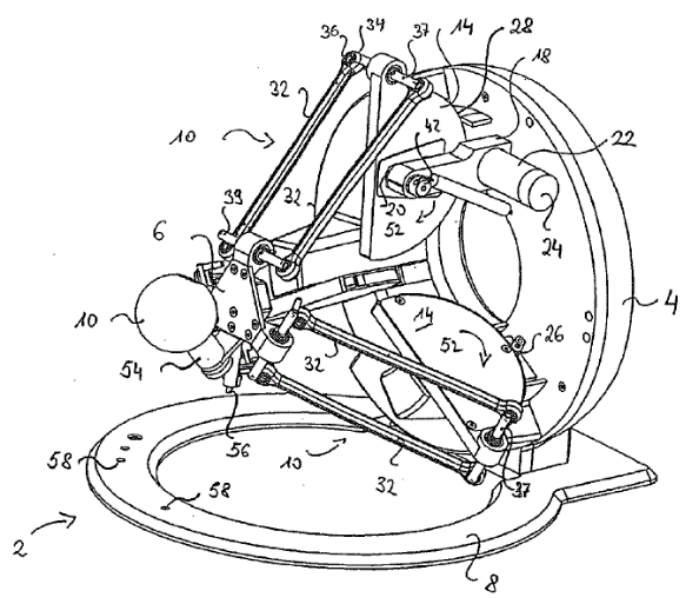

Fig. 4. Omega.3 haptic interface [10] 


\section{Complete-haptic devices}

The first feedback interface was integrated in a robotic tele-operation system for nuclear environments developed by Goertz at Argonne National Laboratories [09]. Since then, different commercial force feedback interfaces have been developed. One simple and inexpensive device is the Falcon 3D from NovInt. It was built for the gaming industry and it has a Delta configuration. A much more complex, reliable and expensive device is the Omega.3 (Figure 4) interface developed by Force Dimension [10]. Built around a parallel kinematic structure, it provides a good haptic effect through a high rendering degree of contact forces. Three similar devices sharing the same platform are commercialized at the moment: Omega.3 (3 active T), Omega.6 (3 active T and 3 passive R) and Omega.7 (3 active $\mathrm{T}$ and 3 passive $\mathrm{R}$ plus 1 active grasping).

There are other similar interfaces. Haption Company (France) proposes the Virtuose 6D Desktop device. It is composed of three articulated branches attached in parallel to a cylindrical handle and it provides 6 degrees of freedom (DoFs), with force-feedback on all 6 DoFs. Immersion Corporation (USA) has patented a haptic pointing device [11]. It consists of several rigid, elongated proximal members. At one end, each proximal member is coupled to a motor, while the other end is connected through an articulated joint to a separate rigid, elongated distal member. Another interesting device, called SHaDe, was developed by researchers from Laval University [12]. The distinctive feature of the SHaDe mechanism is that it has only three rotational DoFs, thereby leading to a simpler design and a more ergonomic interface. Due to a spherical geometry, it has several advantages, namely pure rotation around a point located in the user's palm and precise manipulation by keeping the wrist in a rest position. Despite these advantages, it is interesting to note that this equipment has not been used in industry.

Concluding, the haptic devices based on parallel kinematic structures or on spherical mechanisms can provide good haptic effects, but their workspace is very limited and the cost is high.

A different type of haptic interfaces relies on linkage-based mechanisms, which consist of a pen attached to a robotic arm. The arm can track the position of the pen and it is capable of exerting a force on the tip of this pen. In order to "trick" the user sense of touch, it requires sophisticated hardware and software for determining the proper joint angles and the torques necessary to produce a single point of force on the tip of the pen. Thus, the cost of a high-quality linkage-based force feedback device goes up to tens of thousands of euros.

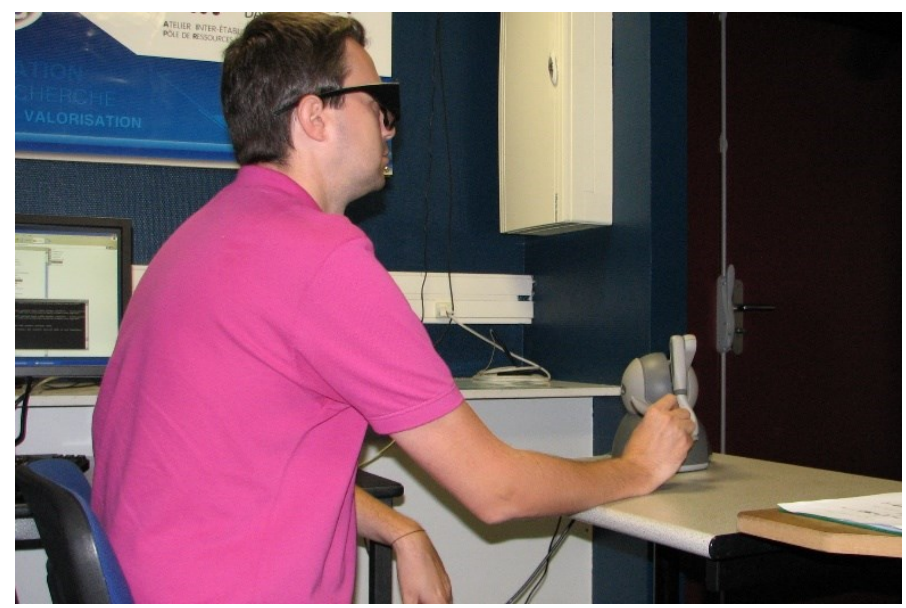

Fig. 5. Researcher using the 3D Systems Touch haptic device 
One well-known and relatively inexpensive device on the market is the 3D Systems Touch (Figure 5). The haptic device is produced by 3D Systems (former Geomagic, former Sensable), offering 6 DoFs positional sensing and 3 DoFs force feedback.

There is also Virtuose 3D Desktop equipment (Haption, France) that provides precision positioning input, high fidelity force-feedback output and a bigger working space for a higher price. It has similar characteristics as 3D Systems Touch X device, being actually its direct competitor, especially on the European VR market. The main difference between the two platforms is, in fact, the Application Programming Interface (API).

The list of high premium haptic devices for general immersive simulations includes three interfaces: Virtuose 6D (Figure 6), Phantom Premium 1.5 (6 DoFs) and Phantom Premium 3.0 (6 DoFs). Virtuose 6D is developed by Haption and it offers a large workspace and force-feedback on all 6 DoFs, especially suited to handling virtual objects at scale 1 [13]. Phantom Premium devices (3D Systems, USA) provide force feedback in three translational DoFs and torque feedback in three rotational DoFs in yaw, pitch and roll directions. The $1.5 / 6$ DoFs device has a range of motion that approximates lower arm movement pivoting at the elbow, while the 3.0/6 DoFs has a range of motion approximating full arm movement pivoting at the shoulder.

Concluding, although all these haptic interfaces offer an excellent haptic feedback, their workspace is still limited and their cost is prohibitive even for medium design companies or research labs.

In addition to the grounded desktop force feedback devices mentioned above, haptic feedback gloves are also available for gaming, simulation and rehabilitation purposes. This type of gloves represents a top version of the digital glove technology. The first feedback glove, called the Rutgers Master, was developed by Burdea and colleagues from Rutgers University [14]. The concept is interesting, but it does not allow complete fist closure due to the placement of the actuators in the palm of the hand. A well-known commercial device is the CyberGrasp manufactured by Cyber Glove Systems (USA). It consists of a cabledriven exoskeleton structure powered by electrical actuators placed on the back of the hand. The design is well fitted, but the glove is relatively heavy - 500 grams, which may lead to user fatigue during use. Another device, more complex, produced by the same company, is the CyberForce. This interface is designed to work with the CyberGrasp exoskeleton in order to offer the possibility, while picking up a virtual object, to feel its weight and inertia, or to feel the resistance opposed by a wall simulated in the VE. This is high quality equipment, but its price is prohibitive for the majority of users.

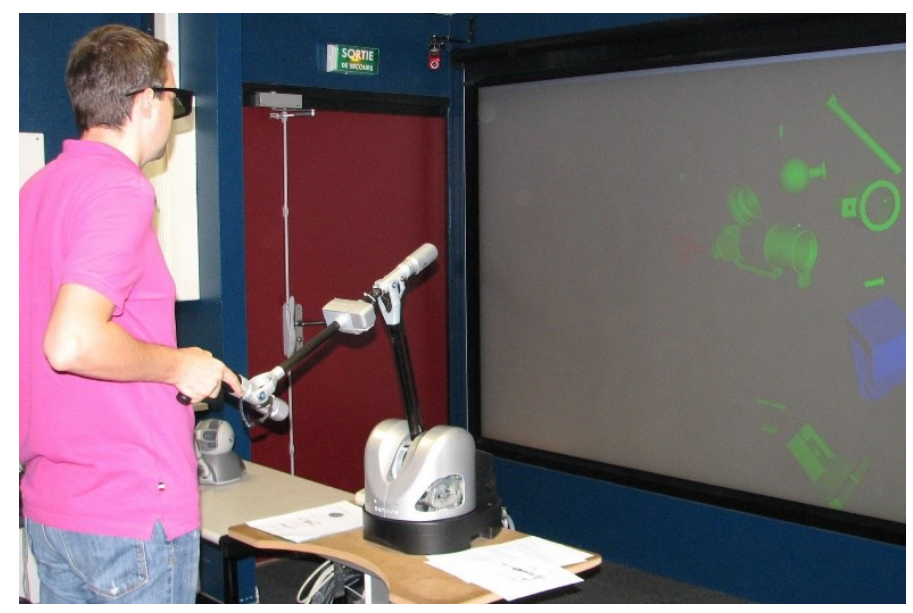

Fig. 6. Researcher using the Haption Virtuose 6D haptic device 
The haptic systems based on robotic exoskeletons represent an alternative to the classical haptic interfaces. These types of systems are currently under research, most of them in a prototype stage. As an example, ABLE is a haptic interface for the hand and the arm developed by Interactive Robotics Unit from CEA Fontenay-aux-Roses (France). It provides up to 7 active DoFs: 4DoFs for the ABLE standard and 3 DoFs for the wrist [15]. Previously, a similar system was proposed by Bionics Lab from University of California. The EXO-UL3 is an exoskeleton initially designed as a human amplifier, but later used also as a haptic device [16]. Another very interesting project is TELESAR V. Tachi Laboratory at Keio University (former University of Tokyo) tries to develop and implement the concept of haptic telexistence by using, as a substitute, an anthropomorphic robot. TELESAR enables the user to bind with a dexterous robot and to experience what the robot can feel from its fingertip when manipulating and touching objects remotely, thus offering a similar haptic sensation.

Concluding, although the exoskeletons haptic interfaces represent an interesting alternative, the technology has some strong limitations in terms of weight, price and working space.

Unlike haptic systems based on mechanical links between elements, the tension-based device created by Sato Makoto [17] and named SPIDAR applies forces directly to the point of contact throughout the tension in the cables. Thus, the limitation on workspace size is completely avoided and, as a result, the system is also scalable. The RIO platform is a type of haptic device ideal for medical simulations, developed by a joint venture between Mako and Immersion companies. It is the first robotic arm system for orthopedic surgery, the application providing patient-specific, three-dimensional modeling for surgery preplanning. The surgeons can use the robotic arm to resurface the knee for implants' placement, the device providing real-time inter-operative visual, tactile and auditory feedback for enhancing the precision of implant positioning. Another platform developed for the medical simulation is Freedom 7S. It is a force feedback device offering high fidelity and operating in 7 DoFs, ideal for master/slave robotics applications in which a sensitive control by a scissors-like handle is required.

Concluding, the haptic platforms developed for medical simulation and operation, are expensive equipment (with a price range situated between 100.000 and 500.000 euro) and offer extremely accurate haptic effects. The customers that might afford such systems are university hospitals and medical research labs.

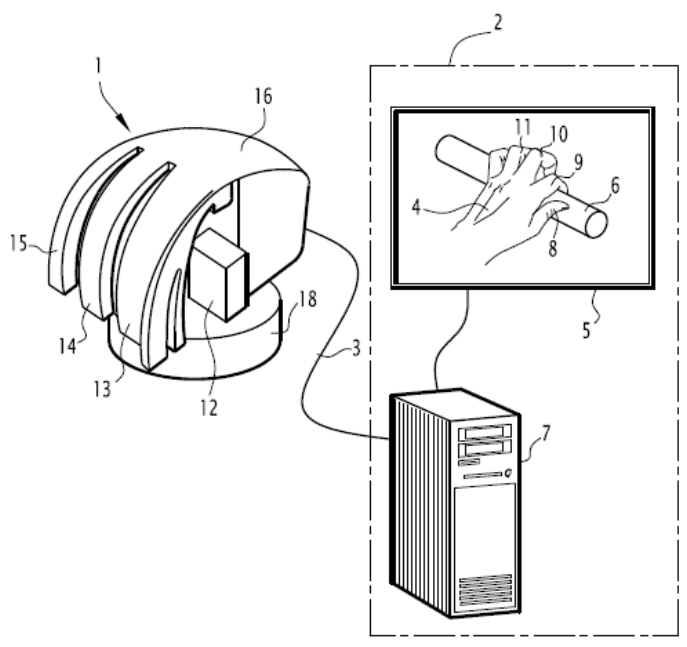

Fig. 7. Hand Navigator device [18] 
Finally, three interesting types of equipment are discussed. The zSpace design laptop, manufactured by Infinite $\mathrm{Z}$ Company (USA), is an immersive and interactive 3D environment for design, communication and entertainment. Pen de Touch, developed by Tachi Laboratory, is a haptic device with a handheld pen-like that allows haptic interactions with VE by providing kinesthetic sensations. Hand Navigator is a novel interaction system dedicated to the manipulations of digital models by using natural hand gestures (Figure 7). It is a peripheral device allowing the user to control a virtual hand with fine dexterity, while sensing passive feedback [18].

\section{Haptics democratization pathway}

Humans are using all senses in their relation with the environment, for communicating and exchanging information with each other. The sense of touch is one important means of communication, intensely used from the very first years of life. Nowadays, immersive simulation environments can provide, to a clear certain extent, the hardware and software solutions for using touch also for communication between man and computer. Thus, the fields of Artificial Intelligence (AI) and Robotics (ROB) have known lately important and, sometimes, even spectacular evolutions in their attempt to copy the way the natural sense of touch is produced, it manifests and it is perceived. For instance, results on the physics of friction for fingerprint-like ridges sliding across textured surfaces may lead the way to tactile robotic sensors [19]. However, we consider that, despite these impressive advances and development potential, the human characteristics and creativity cannot be programmed and cannot have a digital equivalent. Moreover, an exact mimic of human touch is impossible to achieve with the current technology. In this sense, it can be mentioned an interesting research proving how exquisitely sensitive the human finger is, in perceiving different materials, the tactile discrimination extending to the nanoscale [20].

This increasing research focus on using AI and ROB for developing copying the nature/humans raises ethical questions that will need an answer in the foreseeable future, such as: A sense of touch similar to that of humans can and should be developed in robots? Or more generally: Should we even try to mimic humans?!

In the same time, we consider that access to haptics for larger groups of people (users) should be enlarged, by providing them affordable devices and open source software applications. Thus, the field itself and the related applications can develop more rapidly and consistently. We believe that "haptics for everyone" paradigm - introduced by Texas Instruments, can bring only benefits to the field, in the same way the Additive Manufacturing (AM) technology - more precisely, the expiration of several important patents in the field, determined an avalanche of hardware improvements and an increase in the number of applications and manufactured objects. In other words, we consider that value can be added more rapidly to the field, if the haptic technology becomes affordable for larger categories of people.

In our opinion, despite the recognized usefulness of haptics for everyday computer interface, the domain is still under-represented mainly because the existing immersive software is not offering robust functions for real-time simulations, while the commercially available haptic devices that can provide high quality immersion are still relatively expensive.

A complete immersive simulation environment implies a perfect integration between hardware and software. Thus, a set of high-quality equipment to digitally simulate the human senses becomes a main condition. And, as many visual and acoustic high-fidelity solutions already exist, the missing link is currently an affordable and reliable haptic interface. 
This review of the tactile and force feedback devices allows drawing the following set of conclusions:

$\square \quad$ simple tactile devices have affordable prices, but they offer an extremely low haptic feedback;

$\square \quad$ tactile gloves produce better haptic effects, but for a higher price;

$\square \quad$ haptic devices based on parallel kinematic structures provide good haptic effects, but their workspace is very limited and their cost is much higher;

$\square \quad$ arm-based haptic interfaces offer an excellent haptic feedback, but their cost is prohibitive even for medium design companies or research laboratories;

$\square \quad$ exoskeletons haptic interfaces represent an interesting alternative, but the technology has two strong limitations: weight and price;

$\square \quad$ haptic platforms developed for medical simulation and operation offer extremely accurate haptic effects, but their application domain is highly limited.

Therefore, at the moment, the main barrier to the widespread use of haptic devices is the high price. Using the price list from a main VR equipment distributor - Engineering Systems Technologies [21], an average price of a haptic system's hardware and software was computed, and the haptic device price represented more than four-fifths from the whole system price. In this context, a rapid way to decrease the total cost would be to use a device with an affordable price combined with an intelligent immersive simulation environment.

Considering all these elements, there is a strong need of developing a new type of haptic device, based on an innovative modular concept, with the following set of features:

$\square \quad$ its components can be used separately or together;

$\square \quad$ its components can be coupled or decoupled through a special interface similar to robotic gripper interfaces;

$\square \quad$ one component can be used as a 3D mouse by moving the device in space;

$\square \quad$ one component can be used as a 3D pointer to move objects in flying mode;

$\square \quad$ its components generate a tactile feedback when a virtual object is touched;

$\square \quad$ its components contain special sensors for the fingers;

$\square \quad$ one component offers complete haptic feedback.

This new haptic system could represent an innovative and affordable solution for a larger range of potential users: engineers, physicists, trainers, designers, researchers, hobbyists etc.

\section{Conclusions}

Currently, simulation is a key technology in several domains of activity. Different types of simulation, such as: automatic, interactive or immersive, can be applied to 3D models following various objectives: analysis, testing, optimization, training. Related to the particularities of real-time simulations and considering the existence of numerous types of equipment for the video and audio feedback, haptic technology is the missing link at the moment.

In this context, a critical analysis of important haptic equipment, prototypes and commercially available devices, was presented in this paper. Following a general evaluation of existing devices, a critical set of features for a new type of haptic system, based on a modular platform concept, was detailed. This platform could be useful for engineers, physicists, trainers, designers, researchers, as well as for hobbyists.

Further work will be focused on designing the hardware equipment by an interdisciplinary team of researchers based on the concepts presented in this paper.

The work has been partially funded by the EU-RO InnoRESEARCH project under Contract POSDRU/159/1.5/S/132395. 


\section{References}

1. James Jerome Gibson - The senses considered as perceptual systems. Greenwood Publishing Group, Connecticut, U.S.A., ISBN: 978-0313239618 (1983)

2. Gabriel Robles de la Torre - The importance of the sense of touch in virtual and real environments. IEEE MultiMedia Journal vol. 13, no. 3, pp. 24-30 (2006)

3. Grigore C. Burdea - Keynote address: Haptic feedback for virtual reality. Proceedings of the International Workshop on Virtual prototyping, pp. 87-96, Laval, France (1999)

4. Bradford Paley - Three-dimensional mouse with tactile feedback. United States Patent number US.05296871 (1994)

5. Chris Murzanski, Kurt Nielsen, Gary Gordon - Force feedback mechanism for gamepad device. United States Patent number US.07507158 (2009)

6. Jerome Perret, Emmanuel Vander Poorten - Review paper: Commercial haptic gloves. Conference EuroVR, pp. 1-6, London, England (2018)

7. Sean Jason Keller, David Perek, Tristan Thomas Trutna, Garett Andrew Ochs, Nicholas Roy Corson, Raymond King - Actuated tendon pairs in a virtual reality device. United States Application number US.2018.077976 (2018)

8. Zoran Radivojevic, Piers Andrew, Jarkko Saunamaki, Tapani Jokinen - Haptic communication. United States Application number US.2012.062371 (2012)

9. Raymond C. Goertz - A force reflecting positional servo mechanism. Nucleonics vol. 10, part II, pp. 43-45 (1952)

10. Patrick Helmer, Francois Conti, Sebastien Grange, Patrice Rouiller - Active gripper for haptic devices. European Patent Application number EP.01876504 (2008)

11. Douglas Haanpaa, Gary Siebert, Terry Cussen, Kirk Fifer, Mike Dinsmore, Charles Jacobus Haptic pointing device. United States Patent number US.06281651 (2001)

12. Lionel Birglen, Clement Gosselin, Nicolas Pouliot, Bruno Monsarrat, Thierry Laliberte SHaDe: A new 3-DoF haptic device. IEEE Transactions on Robotics and Automation vol. 18, no. 2, pp. 166-175 (2002)

13. Jerome Perret - Haptic trends by Haption: larger, stronger, faster. Conference EuroHaptics, pp. 1-4, Versailles, France (2014)

14. Grigore C. Burdea, Jiachen Zhuang, Edward Roskos, Deborah Silver, Noshir Langrana - A portable dextrous master with force feedback. Journal of Teleoperators and Virtual Environments - Presence vol. 1, no. 1, pp. 18-28 (1992)

15. Philippe Garrec - Design of an anthropomorphic upper limb exoskeleton actuated by ball-screws and cables. UPB Scientific Bulletin D, vol. 72, no. 2, pp. 23-34 (2010)

16. Joel C. Perry, Jacob Rosen, Stephen Burns - Upper-limb powered exoskeleton design. IEEE/ASME Transactions on Mechatronics vol. 12, no. 4, pp. 408-417 (2007)

17. Makoto Sato - SPIDAR and virtual reality. Proceedings of Automation Congress vol. 13, pp. 17$23(2002)$

18. Jean-Rémy Chardonnet, Jean-Claude Leon - Périphérique d'interaction apte à contrôler un élément de toucher et de préhension d'objets virtuels multidimensionnels. Brevet FR.02986342 (2013)

19. C. Mathew Mate, Robert W. Carpick - Materials science: A sense for touch. Nature vol. 480, pp. 189-190 (2011)

20. Lisa Skedung, Martin Arvidsson, Jun Young Chung, Christopher Stafford, Birgitta Berglund, Mark Rutland - Feeling small: exploring the tactile perception limits. Scientific Reports 3: 2617 (2013)

21. Engineering Systems Technologies Company website: https://est-kl.com/products/hapticfeedback.html 